\title{
A CASE REPORT OF TUBERCULOUS PALMAR GANGLION IN IMMUNOCOMPROMISED
}

\author{
Mahaboob Khan', M. Shahana' ${ }^{2}$ K. Vikasna ${ }^{3}$, Ravindranath ${ }^{4}$, K. Subhakar ${ }^{5}$ \\ ${ }^{1}$ Associate Professor, Department of Pulmonary Medicine, Gandhi Medical College. \\ ${ }^{2}$ Assistant Professor, Department of Pulmonary Medicine, Gandhi Medical College. \\ ${ }^{3}$ Resident, Department of Pulmonary Medicine, Osmania Medical College. \\ ${ }^{4}$ Professor, Department of Orthopaedics, Osmania Medical College. \\ 5Professor, Department of Pulmonary Medicine, Osmania Medical College.
}

ABSTRACT: Tuberculous tenosynovitis of flexor tendons of wrist and hand is a rare disease. The incidence of extra-pulmonary tuberculosis including musculoskeletal system has also increased with re-emergence of tuberculosis due to increase in HIV/AIDS cases. We are reporting a case of 45 years old HIV and diabetic male who presented with a swelling and pain in left wrist. Patient was managed by open biopsy with excision of thickened synovium and sample send for histopathological examination. Histopathological report revealed inflammatory lesions with granulomatous epitheloid and giant cells, staining for AFB was negative. ATT was advised to patient for 9 months with regular follow-up. Patient gained full range of motion in wrist last follow-up. The tuberculous tenosynovitis is rare but must be kept in mind as differential diagnosis of chronic tenosynovitis.

KEYWORDS: Tuberculous Tenosynovitis, Flexor Retinaculum, Excision Biopsy, Compound Palmar Ganglion.

HOW TO CITE THIS ARTICLE: Mahaboob Khan, M. Shahana, K. Vikasna, Ravindranath, K. Subhakar. "A Case Report of Tuberculous Palmar Ganglion in Immunocompromised". Journal of Evolution of Medical and Dental Sciences 2015; Vol. 4, Issue 90, November 09; Page: 15593-15595, DOI: 10.14260/jemds/2015/2237.

INTRODUCTION: Tuberculous tenosynovitis of flexor tendons at wrist and palm or compound palmar ganglion is tubercular infection of the tenosynovium of long flexor tendon more common in male and mostly involve dominant limb.[1] Tuberculous tenosynovitis is rare but it is still a leading cause of chronic tendon sheath infection. The route of infection is either direct inoculation or hematogenous dissemination from a primary focus; lungs, lymph nodes, kidney.[1] spine and precipitated by immunosuppression, local/systemic steroid, alcoholism, malnutrition and trauma. ${ }^{[2]}$ Its clinical picture is typical and histopathological studies are carried out for confirmation. The disease can progress; resulting in a gross destruction of surrounding structures.[3] Thus it requires surgical excision without delay. For better prognosis, early recognition and complete surgical excision along with appropriate antitubercular therapy is important.[3]

CASE REPORT: A 45 years old male presented to our OP with the complaints of pain and swelling in the volar aspect of left wrist since 2 months. He is known case of RVD and type II diabetes mellitus on regular medication. Swelling is gradual in onset, progressive in nature.

There is no previous history of any trauma, pulmonary tuberculosis and contact, constitutional symptoms of tuberculosis, rheumatoid arthritis and pain in other joint and lymphadenopathy. On local examination there was swelling $6 \mathrm{~cm} \times 2 \mathrm{~cm}$ in size over volar aspect of left wrist which is mildly tender doughy in consistency,

Financial or Other, Competing Interest: None.

Submission 13-09-2015, Peer Review 14-09-2015,

Acceptance 27-10-2015, Published 09-11-2015.

Corresponding Author:

Dr. Mahaboob Khan,

Gandhi Medical College/Government General and Chest Hospital,

Hyderabad, Telangana State, India.

E-mail:dr_mahaboob@yahoo.com

DOI:10.14260/jemds/2015/2237. compressible extending longitudinally both sides proximal and distal to the flexor retinaculum of the wrist.

There is no distal motor and sensory deficit. Lab investigations were normal .MRI of hand is suggestive of synovial thickening flexor tendons from distal forearm to head of metacarpal level. Edema of hamate bone is present.

An open biopsy was planned and carried out under regional anesthesia was performed. At surgery granulation tissue thickening of synovial membrane and capsule of left tendon was noted. Thorough cleaning and tenosynovectomy was done and specimen sent for histopathology. It revealed inflammatory lesions with granulomatous epitheloid and giant cells.

As per the RNTCP guidelines, category I antitubercular treatment was started. Patient is responding for the treatment and kept under observation.

DISCUSSION: The commonly affected extra-pulmonary sites are lymph nodes, genitourinary tract, bone marrow, CNS and musculoskeletal system which include bones, joint, bursas and tendons or tenosynovium which is $1.3 \%$ of all patients having tuberculosis. ${ }^{1}$

Tubercular tenosynovitis is more common in upper limb as compare to lower limb and commonly involves the flexor or volar site of wrist and hand of dominant limb. 4 The pathogenesis may be direct inoculation or heamatogenous spread from a primary focus in lung, lymph nodes, genitourinary tract and bones. ${ }^{1}$ The onset of tubercular tenosynovitis is characteristically insidious and gradual and the progression of the disease is slow. The symptoms are minimum and very mild and the disease is advance till patient seeks treatment. The symptoms of median nerve compression at carpal tunnel may be there in case of huge and tense swelling. ${ }^{5}$

There are three histopathological stages of disease depending upon duration of disease, resistance of patient and virulence of infecting agent in early stage there is vascular 
granulation tissue formation followed by obliteration of tendon sheath by fibrous tissue fluid accumulation and formation of rice bodies or melon seeds which are fibrinous masses or tubercle made by caseation in last stage there is rupture of tendon sheath, extensive caseation and granulation take place. ${ }^{2}$ The rice bodies are also commonly found in many diseases like SLE, rheumatoid arthritis, seronegative arthritis and osteoarthritis of joint.

The differential diagnosis of compound palmar ganglion includes rheumatoid arthritis, gouty arthritis, pyogenic infection, ganglion [Infected], sarcoidosis, foreign body tenosynovitis, and fungal infection, pigmented villonodular synovitis of tendon sheath, amyloidosis and synovial chondromatosis. The best way to make a final diagnosis is open biopsy and mycobacterial culture of the pathological material but the culture requires few weeks to diagnose which leads to delay in diagnosis and treatment. Combined surgical and medical therapies gives good results and prevent recurrence of disease.

CONCLUSION: Suspicion of compound palmar ganglion in a case of swelling on the volar aspect of hand especially in an immunocompromised hosts helps in early diagnosis and treatment. Hence, preventing the complications.

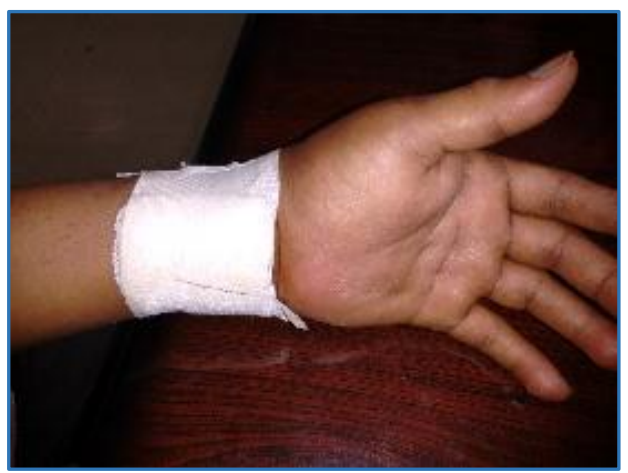

Fig. 1: Post Surgical Picture of Left Hand

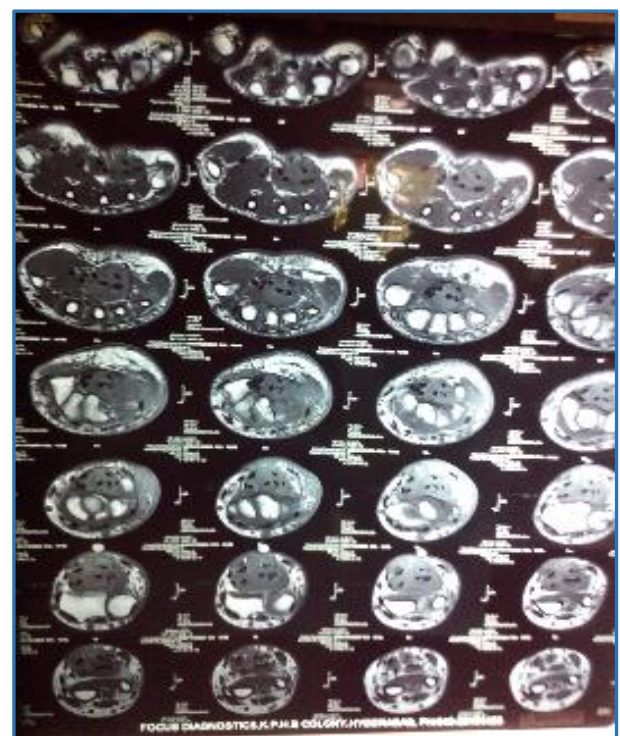

Fig. 3: MRI showing palmar ganglion (2)

\section{REFERENCES:}

1. Seiichi Higuchi, Shinichi Ishihara, Hiroyuki Kobayashi and Taidoh Arai: A mass lesion of the wrist: A rare manifestation of tuberculosis.

[DOI: 10.2169/internalmedicine.47.0495]

2. Hitesh Lall, Suman K Nag, Vijay K Jain, Rahul Khare and Deepak Mittal: Tuberculous extensor tenosynovitis of the wrist with extensor pollicis longus ruptures: a case report. Journal of medical case reports 2009, 3:142.

3. Kumar KA, Kanthimathi B, Krishnamurthy CS, Sujai S; Compound palmar ganglion: A tubercular manifestation of flexor tenosynovitis of the wrist. IJCRI, 2012; 3(2): 28-31.

4. Pei-Hung Shen, Cheng-Mien Chu, Gua-Shu Huang, ShingSheng $\mathrm{Wu}$ and Chain-Her Lee tuberculous tenosynovitis of the flexor tendons of the wrist and hand: J Med Sci 2002;22[5]:227-230

5. Mamoon Rashid, Saad Ur Rehman Sarwar, Ehtesham Ehtesham, Muhammad Zia Ul Islam, Kokab Shah: tubercular tenosynovitis: a cause of carpal tunnel syndrome.JPMA 56:116; 2006.

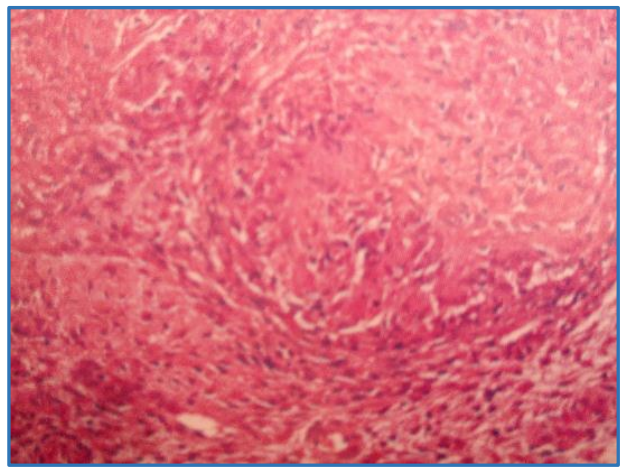

Fig. 2: Histopathological Picture of Granulomatous Lesion

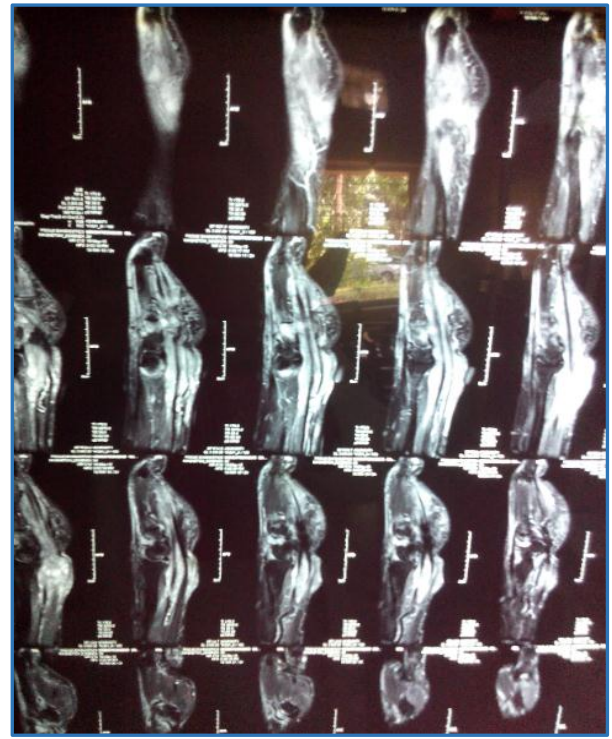

Fig. 4: MRI Showing Palmar Ganglion 


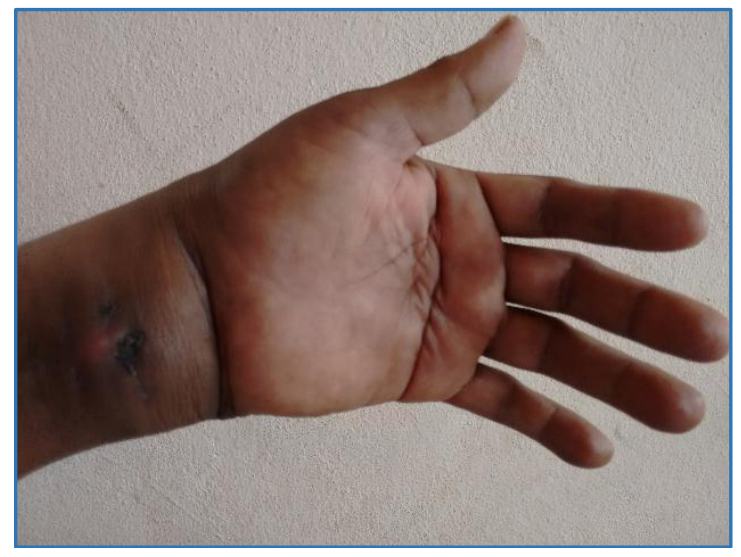

Fig. 5: Swelling in The Volar Aspect of Left Hand 